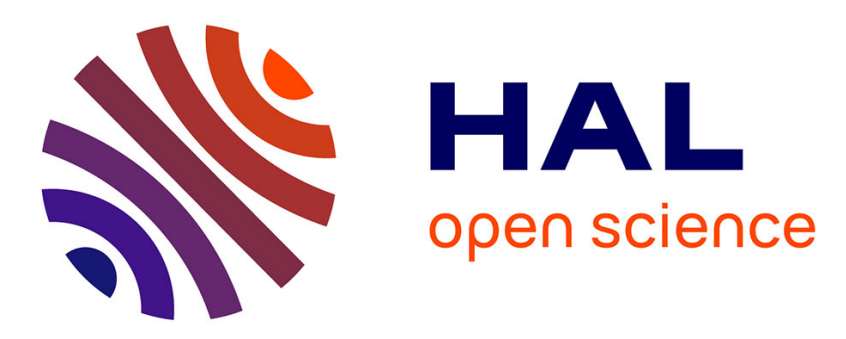

\title{
Stability of distributed delay system with infinite support
}

Yassine Ariba, Frédéric Gouaisbaut, Alexandre Seuret, Kun Liu

\section{To cite this version:}

Yassine Ariba, Frédéric Gouaisbaut, Alexandre Seuret, Kun Liu. Stability of distributed delay system with infinite support. 57th IEEE Conference on Decision and Control (CDC 2018), Dec 2018, Miami,

United States. 10.1109/CDC.2018.8619274 . hal-01962563

\section{HAL Id: hal-01962563 \\ https://hal.laas.fr/hal-01962563}

Submitted on 20 Dec 2018

HAL is a multi-disciplinary open access archive for the deposit and dissemination of scientific research documents, whether they are published or not. The documents may come from teaching and research institutions in France or abroad, or from public or private research centers.
L'archive ouverte pluridisciplinaire HAL, est destinée au dépôt et à la diffusion de documents scientifiques de niveau recherche, publiés ou non, émanant des établissements d'enseignement et de recherche français ou étrangers, des laboratoires publics ou privés. 


\title{
Stability of distributed delay system with infinite support
}

\author{
Yassine Ariba, Frédéric Gouaisbaut, Alexandre Seuret and Kun Liu
}

\begin{abstract}
This paper is devoted to the stability analysis of infinite distributed delay systems. The basic idea is to model the original time-delay system into an interconnected feedback system in order to use robust analysis and especially quadratic separation. This approach has been widely used to study classical pointwise time-delay system. The stability analysis is performed by introducing new quadratic inequalities based on Laguerre polynomials particularly well fitted to deal with infinite distributed delay. It allows to develop new stability results which accuracy depends on the chosen polynomial degree.
\end{abstract}

\section{INTRODUCTION}

For several years, we have experienced a renewal in the study of the stability and stabilisation of delay systems [20]. Indeed, the study of stability is mainly carried out by using a Lyapunov-Krasovskii functional whose parameters are determined by means of a convex optimization scheme. For several years now, these functionals have become very efficient thanks to the relevant choices of their structure [21] which has become more complex and incorporates new states related to the partitioning of the delay [7], integral of states [5] or the projection of the infinite-dimensional delay state on an orthogonal polynomial basis [16]. This last feature also allows the use of very precise quadratic inequalities leading to very efficient LMI criteria. These inequalities, called Bessel inequalities, are all primarily based on the relevant choice of an inner product in $L_{2}$ and the use of a polynomial orthogonal basis. Thus, Legendre polynomials have been used to develop stability criteria for pointwise delay systems in a Lyapunov-Krasovskii functionals framework but also in a robust analysis setup [1]. Notice that these inequalities include the inequalities of Jensen [8] and Wirtinger [15] widely used in the literature on delay systems. Concerning distributed time-delay systems with a finite support, several papers have been devoted to the construction of Lyapunov functionals [3], [4], [5] (and references therein). The methodology involving orthogonal Legendre polynomials has also led to results reducing the conservatism of Lyapunov methods and keeping a relatively low numerical complexity [17], [6].

Attempts to study systems with distributed delay with an infinite support are more scarce. For instance, frequency approaches have been developed to study the stability of

Y. Ariba, F. Gouaisbaut, A. Seuret are with LAAS - CNRS, Université de Toulouse, CNRS, UPS, France e-mail: (yariba,fgouaisb,aseuret,@laas.fr).

Y. Ariba is also with the school of engineering Icam, Toulouse, France.

Kun Liu is with the Key Laboratory of complex system control and decision, School of Automation, Beijing Institute of Technology, Beijing 100081, China mail: (kunliubit@bit.edu.cn).

This work is supported by the ANR project SCIDiS contract number 15-CE23-0014. the traffic flow dynamics [13] or linear systems with gamma distributed delays [18]. These systems have also been studied under the angle of an extension of the functional [19]. The interest of such methods is mainly the development of new quadratic integral inequalities with infinite support of integration. This has led to the construction of new Lyapunov functionals, which, combined with optimization algorithms, allowed at least on examples, to obtain very encouraging results [19]. It should be noted that this approach has recently been extended by using terms that depend on a double integral in order to significantly improve the results [11].

The paper's objective is to study this class of delay systems using robust analysis, a method that has already led to the development of robust stability conditions for constant delay systems (pointwise or distributed). We focus more specifically on the quadratic separation approach whose principle is to model the distributed delay system as a closed loop system between an uncertain dynamical system and a linear transformation. The novelty of the approach lies in the use of a new inner product taking into account the infinite support of the distributed delay system. The kernel of the distributed part is thus, within this framework, approximated by a sum of Laguerre polynomials which are orthogonal for the selected inner product. Notice that the approximation error is also taken into account in the rewriting of the original model. A new comparison model is therefore obtained, in which the uncertainty set, gathering the delay elements, can be precisely described by using a Bessel-Laguerre inequality. Using the quadratic separation theorem, a sequence of LMIs tests, in which conservatism decreases as the degree of Laguerre polynomials increases is then proposed. This approach has to be viewed as the robust counterpart of [12], where Laguerre polynomials have been also used in a Lyapunov framework.

Notations: Throughout the paper, the following notations are used. The sets $\mathbb{N}, \mathbb{R}, \mathbb{C}$ represent the set of non negative integer numbers, of real and complex number, respectively. The set $\mathbb{R}^{n \times m}$ is the set of matrices of $n$ rows and $m$ columns. $1_{n}$ and $0_{m \times n}$ denote respectively the identity matrix of size $n$ and null matrix of size $m \times n$. If the context allows it, the dimensions of these matrices will be omitted. For two symmetric matrices, $A$ and $B, A>(\geq) B$ means that $A-B$ is (semi-) positive definite. $A^{T}$ denotes the transpose of $A . A^{\perp}$ stands for a full rank matrix whose columns span the null-space of $A$, i.e. $A A^{\perp}=0 . \operatorname{diag}(A, B)$ stands for the block diagonal matrix $\operatorname{diag}(A, B)=\left[\begin{array}{ll}A & 0 \\ 0 & B\end{array}\right]=\left[\begin{array}{ll}{ }^{A} & \\ B\end{array}\right]$. The notation $A^{*}$ represents the conjugate transpose of the complex matrix $A . L_{2}$ can be viewed as the space of square integrable functions with a finite energy: $\|f\|^{2}=$ 
$\int_{0}^{\infty}|f(t)|^{2} d t . H_{2}$ is the function space which contains the Laplace transform of signals in $L_{2} . H_{\infty}$ is the set of all functions $F(s)$ such that $\sup _{R e(s) \geq 0} \bar{\sigma}(F(s)) \leq \infty$, where $\bar{\sigma}$ stands for the maximum singular value.

\section{PROBlem STATEMENT}

Let us consider a distributed delay system of the form:

$$
\dot{x}(t)=A x(t)+A_{d} \int_{0}^{\infty} K(\theta) x(t-\theta-h) d \theta,
$$

where $x(t) \in \mathbb{R}^{n}$ is the state vector and is assumed to belong to $L_{2}, A$ and $A_{d} \in \mathbb{R}^{n \times n}$ are constant matrices. The constant delay $h$ is unknown but belongs to an interval $\left[h_{\min }, h_{\max }\right]$. The initial condition is given by $\phi \in C^{1}(-\infty, 0]$, where $C^{1}(-\infty, 0]$ denotes the space of continuously differentiable functions $\phi:(-\infty, 0] \rightarrow R^{n}$ with the norm

$$
\|\phi\|_{C^{1}}=\sup _{s \in(-\infty, 0]}|\phi(s)|+\sup _{s \in(-\infty, 0]}|\dot{\phi}(s)| .
$$

The kernel function $K$ is assumed to be a scalar function of the form

$$
K(\theta)=f(\theta) e^{-\alpha \theta}
$$

where the scalar function $f$ is continuous and verifies $\int_{0}^{\infty} f(\theta) e^{-\alpha \theta} d \theta<\infty$ with $\alpha$ a positive scalar. Following [19], this specific inequality allows us to ensure that the integral in the distributed delay part is convergent. Taking the Laplace transform of (1), we obtain:

$$
\left(s 1_{n}-A-A_{d} \int_{0}^{\infty} K(\theta) e^{-s \theta} e^{-s h} d \theta\right) x(s)=0 .
$$

We aim at assessing the stability of system (1) for a given kernel function $K$ and a given maximal allowable constant gap delay $h_{\max }$. More precisely, following [9], the system is asymptotically stable for all delays $h$ belonging $\left[0, h_{\max }\right]$ if and only if

$$
\begin{aligned}
& \forall h \in\left[0, h_{\text {max }}\right], \forall s \in \mathbb{C}, R e(s) \geq 0, \\
& \operatorname{det}\left(s 1_{n}-A-A_{d} \int_{0}^{\infty} K(\theta) e^{-s \theta} e^{-s h} d \theta\right) \neq 0 .
\end{aligned}
$$

This paper proposes the use of a classical robust analysis approach to address this problem. Firstly, we will model the given system as an uncertain system [22]. Then in a second stage, the stability of the uncertain system will be studied using the Quadratic Separation approach [10], [14], recalled in the following subsection.

\section{PRELIMINARIES}

\section{A. Quadratic separation Approach}

The quadratic separation principle is a specific approach to the robust analysis/control [10], [14]. It consists of analyzing the stability of a specific feedback system as illustrated in Figure 1. This latter is composed of a linear singular system, with two real constant matrices $\mathcal{E}$ and $\mathcal{A}$, connected to a linear operator, with a complex matrix $\nabla$. Basically, this operator encompasses the uncertain feature of the system, which can stem from uncertain parameters, nonlinear dynamics, delay dynamics [2]. It is assumed to belong to an uncertain set $\mathbb{W}$. Hence, the methodological principle relies on the modelling of the system under study so as to obtain an interconnected system as shown in Figure 1. Then a stability result, stated in Theorem 1, is applied to derive a wellposedness condition for the original system. This last condition allows also to prove the robust stability if the uncertain set $\mathbb{W}$ is well chosen.

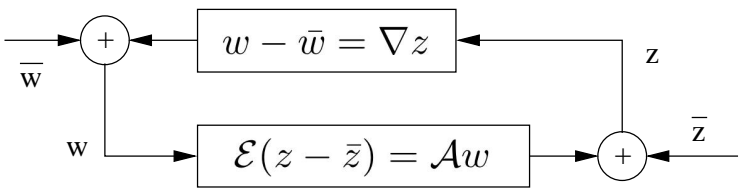

Fig. 1. Feedback system.

Theorem 1 ([14]): The uncertain feedback system of Figure 1 is well-posed and stable if and only if there exists a Hermitian matrix $\Theta=\Theta^{*}$ satisfying both conditions

$$
\begin{gathered}
{\left[\begin{array}{cc}
\mathcal{E} & -\mathcal{A}
\end{array}\right]^{\perp *} \Theta\left[\begin{array}{ll}
\mathcal{E} & -\mathcal{A}
\end{array}\right]^{\perp}>0,} \\
{\left[\begin{array}{c}
1 \\
\nabla
\end{array}\right]^{*} \Theta\left[\begin{array}{c}
1 \\
\nabla
\end{array}\right] \leq 0 \quad, \quad \forall \nabla \in \mathbb{W} .}
\end{gathered}
$$

In practice, inequality (3) is defined at the modelling step, according to the choice of operators in the feedback system. Then, the second inequality (2) is studied so as to design a stability condition to be tested.

\section{B. Laguerre Polynomials}

In [16], a generalized integral inequality has been introduced thanks to the use of the Bessel inequality together with the orthogonal sequence of Legendre polynomials. This formulation was adapted to the situation of distributed delay on finite support [17], but cannot be directly extended to the case of infinite support for the distributed delay term. Therefore, there is a need to formulate a new Bessel-like inequality dedicated to this situation, which is made possible through the use of Laguerre polynomials.

We define by $\mathcal{M}$ the vector space of complex valued square integrable functions on $[0, \infty)$ with an exponential weight. For any functions in $\mathcal{M}$, we define the Hermitian inner product useful for deriving some stability tests:

$$
\langle x, y\rangle=\int_{0}^{\infty} x(\theta) \bar{y}(\theta) e^{-\alpha \theta} d u
$$

where $x$ and $y$ belong to $\mathcal{M}$. As in the definition of the kernel $K(\theta)$, the same coefficient $\alpha$ is used to define this specific inner product. Associated to this inner product, the Laguerre polynomials are also introduced.

Definition 1 (Laguerre polynomials): The Laguerre polynomials are defined by

$$
\forall k \in \mathbb{N}, \quad L_{k}(u)=\frac{e^{u}}{k !} \frac{d^{k}}{d u^{k}}\left[e^{-u} u^{k}\right],
$$


with $L_{k}(0)=1$, for all $k \in \mathbb{N}$. These polynomials are orthogonal with respect to the following inner product, $\forall(k, l) \in \mathbb{N}^{2}$,

$$
\int_{0}^{\infty} L_{k}(u) L_{l}(u) e^{-u} d u= \begin{cases}0, & k \neq l \\ 1, & k=l .\end{cases}
$$

In order to adapt the Laguerre polynomials to the inner product (4), the polynomial definition is slightly modified by a change of variable $u=\alpha \theta$. The new polynomials considered in this paper are then of the form:

$$
\forall k \in \mathbb{N}, \quad L_{k}(\theta)=\frac{e^{\alpha \theta}}{k !} \frac{d^{k}}{d \theta^{k}}\left[e^{-\alpha \theta} \theta^{k}\right] .
$$

Notice that in this case, we have:

$$
\left\langle L_{k}, L_{l}\right\rangle=\frac{1}{\alpha} \kappa_{k l},
$$

where $\kappa_{k l}$ is the Kronecker function. We now propose a first result that will be used later in the modelling part.

Lemma 1: The derivative of polynomials $L_{k}$, defined in (6), can be expressed as a recurrence relation:

$$
\left\{\begin{aligned}
\frac{d}{d \theta} L_{k}(\theta) & =-\alpha L_{k-1}(\theta)+\frac{d}{d \theta} L_{k-1}(\theta), \\
L_{0}(\theta) & =1 .
\end{aligned}\right.
$$

Hence, we also have

$$
\frac{d}{d \theta} L_{k}(\theta)=-\alpha \sum_{l=0}^{l-1} L_{l}(\theta) .
$$

Proof: Let us express the derivative of (6):

$$
\begin{aligned}
\frac{d}{d \theta} L_{k}(\theta)= & \frac{d}{d \theta}\left(\frac{e^{\alpha \theta}}{k !} \frac{d^{k}}{d \theta^{k}}\left[e^{-\alpha \theta} \theta^{k}\right]\right) \\
\frac{d}{d \theta} L_{k}(\theta)= & \frac{e^{\alpha \theta}}{(k-1) ! \frac{d^{k}}{d \theta^{k}}\left[e^{-\alpha \theta} \theta^{k-1}\right]} \\
= & \underbrace{\frac{e^{\alpha \theta}}{(k-1) !} \frac{d^{k-1}}{d \theta^{k-1}}\left[-\alpha e^{-\alpha \theta} \theta^{k-1}\right]}_{-\alpha L_{k-1}} \\
& +\underbrace{\frac{e^{\alpha \theta}}{(k-2) !} \frac{d^{k-1}}{d \theta^{k-1}}\left[e^{-\alpha \theta} \theta^{k-2}\right]}_{\frac{d}{d \theta} L_{k-1}},
\end{aligned}
$$

which concludes the proof.

\section{STABILITY ANALYSIS}

\section{A. Modeling of the original system}

A first step is to model the original system into an interconnected uncertain comparison system whose stability ensures the stability of the original time delay system. In order to benefit from the Laguerre polynomials properties, function $f$ is reworded as

$$
f(\theta)=\sum_{k=0}^{r} a_{k} L_{k}(\theta)+g_{r}(\theta)
$$

where $r$ is the degree of the polynomial and the coefficients $a_{i}$ are computed with the orthogonal projection of the function on the associated Laguerre polynomials,

$$
a_{k}=\frac{\left\langle f, L_{k}\right\rangle}{\left\langle L_{k}, L_{k}\right\rangle}, \quad \forall k=0, \ldots, r .
$$

The function $g_{r}$ is called the remainder at order $r$. The original time-delay system (1) can then be rewritten as:

$$
\begin{aligned}
\dot{x}(t)= & A x(t)+\sum_{k=0}^{r} A_{d k} \int_{0}^{\infty} L_{k}(\theta) e^{-\alpha \theta} x(t-\theta-h) d \theta \\
& +A_{d} \int_{0}^{\infty} g_{r}(\theta) e^{-\alpha \theta} x(t-\theta-h) d \theta
\end{aligned}
$$

with $A_{d k}=a_{k} A_{d}$. Let us define new extra-states as:

$$
\begin{aligned}
& x_{k}(t)=\int_{0}^{\infty} L_{k}(\theta) e^{-\alpha \theta} x(t-\theta-h) d \theta, \quad \forall k=0, \ldots, r \\
& x_{g_{r}}(t)=\int_{0}^{\infty} g_{r}(\theta) e^{-\alpha \theta} x(t-\theta-h) d \theta .
\end{aligned}
$$

The dynamics of the augmented states $x_{k}$ is written as:

$$
\begin{aligned}
\dot{x}_{k}(t) & =\int_{0}^{\infty} L_{k}(\theta) e^{-\alpha \theta} \frac{d}{d t} x(t-\theta-h) d \theta, \\
& =-\int_{0}^{\infty} L_{k}(\theta) e^{-\alpha \theta} \frac{d}{d \theta} x(t-\theta-h) d \theta .
\end{aligned}
$$

An integration by parts and the use of Lemma 1 lead to

$$
\dot{x}_{k}(t)=x(t-h)-\alpha \sum_{l=0}^{k} x_{l}(t) .
$$

This auxiliary dynamic is then appended to the initial system (7) to obtain the following extended system:

$$
\left\{\begin{aligned}
\dot{x}(t) & =A x(t)+\sum_{k=0}^{r} A_{d k} x_{k}(t)+A_{d} x_{g_{r}}(t), \\
\dot{x}_{k}(t) & =x(t-h)-\alpha \sum_{l=0}^{k} x_{l}(t), \quad k=0, \ldots, r .
\end{aligned}\right.
$$

with the particular initial conditions

$$
\left\{\begin{array}{l}
x(s)=\phi(s), \quad s \in(-\infty, 0], \\
x_{k}(0)=\int_{0}^{\infty} L_{k}(\theta) e^{-\alpha \theta} \phi(-\theta-h) d \theta, \quad \forall k=0, \ldots, r .
\end{array}\right.
$$

It is worth noting that the augmented system (8), omitting the constraints on the initial conditions of the $x_{k}$ 's, represents a wider class of system than the original distributed delay system (1). However, the objective of the latter developments is to assess stability of the (8) with the constrained initial conditions in order to guarantee the stability of the initial system (1).

Notice that in the Laplace domain, the original dynamical system can be written as

$$
\begin{aligned}
s x(s)= & A x(s)+\sum_{k=0}^{r} A_{d k} \underbrace{\int_{0}^{\infty} L_{k}(\theta) e^{-s \theta} e^{-\alpha \theta} d \theta}_{\delta_{k}(s)} e^{-s h} x(s) \\
& +A_{d} \underbrace{\int_{0}^{\infty} g_{r}(\theta) e^{-s \theta} e^{-\alpha \theta} d \theta}_{\delta_{g_{r}}(s)} e^{-s h} x(s),
\end{aligned}
$$


emphasizing the relationship between the infinite part of the system and the extra states $x_{k}$ and $x_{g_{r}}$. To this end, we introduce a set of transfer functions as

$$
\left\{\begin{array}{l}
\delta_{k}(s)=\int_{0}^{\infty} L_{k}(\theta) e^{-s \theta} e^{-\alpha \theta} d \theta, \quad k=0, \ldots, r, \\
\delta_{g_{r}}(s)=\int_{0}^{\infty} g_{r}(\theta) e^{-s \theta} e^{-\alpha \theta} d \theta .
\end{array}\right.
$$

At this stage, the extended time delay system (8) can be modeled as an uncertain interconnected system. It can be done by considering the following complex valued matrix

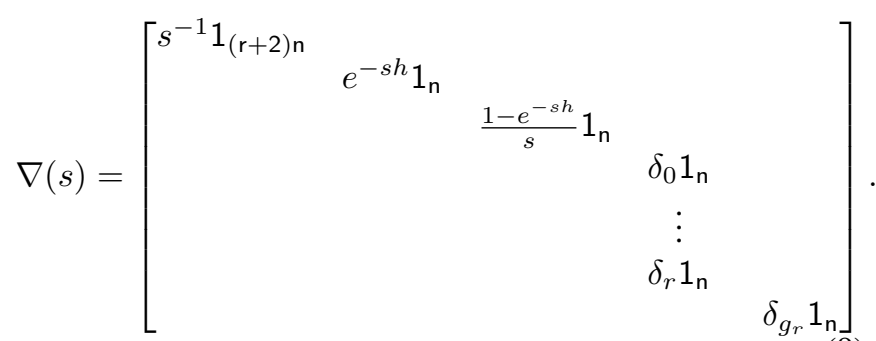

interconnecting the signals

$$
w(t)=\left[\begin{array}{c}
x(t) \\
x_{0}(t) \\
\vdots \\
x_{r}(t) \\
\hline x(t-h) \\
\hline x(t)-x(t-h) \\
x_{0}(t) \\
x_{r}(t)
\end{array} ;(t)=\left[\begin{array}{c}
\dot{x}(t) \\
\dot{x}_{0}(t) \\
\vdots \\
\dot{x}_{r}(t) \\
\hline x(t) \\
\hline x_{g}(t)
\end{array}\right]\left[\begin{array}{c}
\dot{x}(t) \\
\hline x(t-h) \\
\end{array}\right]\right.
$$

with $W(s)=\nabla(s) Z(s)$, in the Laplace domain. Using the definition of the augmented system (8), a linear transformation of the form $\mathcal{E} Z(s)=\mathcal{A} W(s)$ linking $W(s)$ and $Z(s)$ can be built with

$$
\begin{gathered}
\mathcal{E}=\left[\begin{array}{c}
1_{(\mathrm{r}+6) \mathrm{n}} \\
0(\mathrm{r}+2) \mathrm{n} \times(\mathrm{r}+6) \mathrm{n}
\end{array}\right], \\
\mathcal{A}=\left[\begin{array}{cccccc}
A & \bar{A}_{d} & 0 & 0 & 0 & A_{d} \\
0 & U & E & 0 & 0 & 0 \\
1 & 0 & 0 & 0 & 0 & 0 \\
A & \bar{A}_{d} & 0 & 0 & 0 & A_{d} \\
0 & 0 & 1 & 0 & 0 & 0 \\
0 & 0 & 1 & 0 & 0 & 0 \\
1 & 0 & -1 & -1 & 0 & 0 \\
0 & 1 & 0 & 0 & -1 & 0
\end{array}\right],
\end{gathered}
$$

and

$$
\begin{gathered}
E=\left[\begin{array}{c}
1 \\
1 \\
\vdots \\
1
\end{array}\right], U=\left[\begin{array}{cccc}
-\alpha 1 & 0 & \ldots & 0 \\
-\alpha 1 & -\alpha 1 & & 0 \\
\vdots & & \ddots & \\
-\alpha 1 & -\alpha 1 & \ldots & -\alpha 1
\end{array}\right] \\
\bar{A}_{d}=\left[\begin{array}{llll}
A_{d 0} & A_{d 1} & \ldots & A_{d r}
\end{array}\right]
\end{gathered}
$$

\section{B. Construction of quadratic constraints}

The original time-delay has been modeled as an interconnected system depicted in Figure 1 and the next step is to find a so-called separator $\Theta$ such the inequality (3) is satisfied. Since, the uncertain complex valued matrix $\nabla(s)$ is a block diagonal matrix, a separator of the same structure is developed, that is a separator for each component of $\nabla(s)$. The next Lemmas recall some separators classically used in the literature of robust analysis of time-delay systems [2].

Lemma 2: A quadratic constraint for $s^{-1}$ is given by the following inequality for any positive definite matrix $P$ in $\mathbb{R}^{n \times n}$,

$$
\begin{gathered}
\forall s \in \mathbb{C}, \operatorname{Re}(s) \geq 0, s \neq 0, \\
{\left[\begin{array}{c}
1_{\mathrm{n}} \\
s^{-1} 1_{\mathrm{n}}
\end{array}\right]^{*}\left[\begin{array}{cc}
0 & -P \\
-P & 0
\end{array}\right]\left[\begin{array}{c}
1_{\mathrm{n}} \\
s^{-1} 1_{\mathrm{n}}
\end{array}\right] \leq 0 .}
\end{gathered}
$$

Lemma 3: A quadratic constraint for $e^{-h s}$ is given by the following inequality for any positive definite matrix $Q_{1}$ in $\mathbb{R}^{n \times n}$,

$$
\forall s \in \mathbb{C}, \operatorname{Re}(s) \geq 0,
$$

$$
\left[\begin{array}{c}
1_{\mathrm{n}} \\
e^{-h s} 1_{\mathrm{n}}
\end{array}\right]^{*}\left[\begin{array}{cc}
-Q_{1} & 0 \\
0 & Q_{1}
\end{array}\right]\left[\begin{array}{c}
1_{\mathrm{n}} \\
e^{-h s} 1_{\mathrm{n}}
\end{array}\right] \leq 0 .
$$

Lemma 4: A quadratic constraint for $\mu=\left(1-e^{-h s}\right) s^{-1}$ is given by the following inequality for any positive definite matrix $Q_{2}$ in $\mathbb{R}^{n \times n}$,

$$
\begin{gathered}
\forall s \in \mathbb{C}, \operatorname{Re}(s) \geq 0 \\
{\left[\begin{array}{c}
1_{\mathrm{n}} \\
\mu 1_{\mathrm{n}}
\end{array}\right]^{*}\left[\begin{array}{cc}
-h_{\max }^{2} Q_{2} & 0 \\
0 & Q_{2}
\end{array}\right]\left[\begin{array}{c}
1_{\mathrm{n}} \\
\mu 1_{\mathrm{n}}
\end{array}\right] \leq 0 .}
\end{gathered}
$$

Proofs of Lemma 2, 3 and 4 can be found in [2]. The next Lemma is dedicated to the design of a separator for the new operator $\left[\begin{array}{lll}\delta_{0} 1_{\mathrm{n}} & \ldots & \delta_{r} 1_{\mathrm{n}}\end{array}\right]^{T}$.

Lemma 5: A quadratic constraint for $\left[\begin{array}{lll}\delta_{0} 1_{\mathrm{n}} & \ldots & \delta_{r} 1_{\mathrm{n}}\end{array}\right]^{T}$ is given by the following inequality for any positive definite matrix $R_{1}$ in $\mathbb{R}^{n \times n}$,

$$
\left[\begin{array}{c}
1_{\mathrm{n}} \\
\delta_{0} 1_{\mathrm{n}} \\
\vdots \\
\delta_{r} 1_{\mathrm{n}}
\end{array}\right]^{T}\left[\begin{array}{cccc}
-\frac{1}{\alpha^{2}} R_{1} & 0 & \cdots & 0 \\
0 & R_{1} & & \\
\vdots & & \ddots & \\
0 & & & R_{1}
\end{array}\right]\left[\begin{array}{c}
1_{\mathrm{n}} \\
\delta_{0} 1_{\mathrm{n}} \\
\vdots \\
\delta_{r} 1_{\mathrm{n}}
\end{array}\right] \leq 0 .
$$

Proof: Let us apply the Bessel inequality to the delay transfer function $e^{-s \theta}$ with the orthonormal sequence $\left\{L_{0}, \ldots, \sqrt{\alpha} L_{k}, \ldots, \sqrt{\alpha} L_{r}\right\}$ :

$$
\begin{aligned}
\left\langle e^{-s \theta}, e^{-s \theta}\right\rangle & \geq \sum_{k=0}^{r}\left|\left\langle e^{-s \theta}, \sqrt{\alpha} L_{k}\right\rangle\right|^{2} \\
& \geq \sum_{k=0}^{r} \alpha \delta_{k} \delta_{k}^{*} .
\end{aligned}
$$

Since $\left\langle e^{-s \theta}, e^{-s \theta}\right\rangle$ is bounded by $1 / \alpha$, we have

$$
\begin{gathered}
\sum_{k=0}^{r} \alpha \delta_{k} \delta_{k}^{*} \leq \frac{1}{\alpha}, \\
\sum_{k=0}^{r} \delta_{k} \delta_{k}^{*} \leq \frac{1}{\alpha^{2}} .
\end{gathered}
$$


This last inequality can then be directly extended to $\left[\begin{array}{lll}\delta_{0} 1_{\mathrm{n}} & \ldots & \delta_{r} 1_{\mathrm{n}}\end{array}\right]^{T}$ using a matrix $R_{1}>0$ following the approach proposed in [2], which concludes the proof.

The next step is therefore to propose a separator for $\delta_{g_{r}}$.

Lemma 6: A quadratic constraint for $\delta_{g_{r}}$ is given by the following inequality for any positive definite matrix $R_{2}$ in $\mathbb{R}^{n \times n}$,

$$
\begin{gathered}
\forall s \in \mathbb{C}, \operatorname{Re}(s) \geq 0 \\
{\left[\begin{array}{c}
1_{\mathrm{n}} \\
\delta_{g_{r}} 1_{\mathrm{n}}
\end{array}\right]^{*}\left[\begin{array}{cc}
-\lambda_{r} R_{2} & 0 \\
0 & R_{2}
\end{array}\right]\left[\begin{array}{c}
1_{\mathrm{n}} \\
\delta_{g_{r}} 1_{\mathrm{n}}
\end{array}\right] \leq 0,}
\end{gathered}
$$

with

$$
\lambda_{r}=\left(\int_{0}^{\infty}|g(\theta)| e^{-\alpha \theta} d \theta\right)^{2}
$$

Proof: Let us express the norm of $\delta_{g}$ :

$$
\begin{aligned}
\delta_{g_{r}} \delta_{g_{r}}^{*} & =\left|\int_{0}^{\infty} g_{r}(\theta) e^{-\alpha \theta} e^{-s \theta} d \theta\right|^{2} \\
& \leq\left(\int_{0}^{\infty}\left|g_{r}(\theta) e^{-\alpha \theta} e^{-s \theta}\right| d \theta\right)^{2} \\
& \leq\left(\int_{0}^{\infty}\left|g_{r}(\theta)\right| e^{-\alpha \theta} d \theta\right)^{2}=\lambda_{r} .
\end{aligned}
$$

This last inequality can then be extended straightforwardly to $\delta_{g_{r}} 1_{\mathrm{n}}$ introducing a weighting matrix $R_{2}$ [2].

Finally, concatenating all the separators for each operator, we proposed the following separator for $\nabla(s)$.

Lemma 7: Assume there exist positive definite matrices $P, Q_{1}, Q_{2}, R_{1}, R_{2}$, then a matrix $\Theta$ of the form

$$
\Theta=\left[\begin{array}{ll}
\Theta_{11} & \Theta_{12} \\
\Theta_{12}^{T} & \Theta_{22}
\end{array}\right]
$$

with

$$
\begin{aligned}
& \Theta_{11}=\operatorname{diag}\left(0,-Q_{1},-h_{\text {max }}^{2} Q_{2},-\frac{1}{\alpha^{2}} R_{1},-\lambda_{r} R_{2}\right), \\
& \Theta_{12}=\operatorname{diag}(-P, 0,0,0,0), \\
& \Theta_{22}=\operatorname{diag}\left(0, Q_{1}, Q_{2}, R_{1}, \ldots, R_{1}, R_{2}\right),
\end{aligned}
$$

is a separator for $\nabla(s)$ defined by equation (9). The inequality (3) is then fulfilled, for all $s \in \mathbb{C}, \operatorname{Re}(s) \geq 0, s \neq 0$.

Proof: The proof is omitted because it is a direct combination of previous Lemmas.

\section{Stability results}

Having modeled the system (1) as an uncertain interconnected system and having characterized the uncertainty $\nabla(s)$ with Lemma 7, a stability theorem is proposed.

Theorem 2: For a given constant $h_{\max }$, if there exist positive definite matrices $P \in \mathbb{R}^{n(r+2) \times n(r+2)}, Q_{1}, Q_{2}, R_{1}$ and $R_{2} \in \mathbb{R}^{n \times n}$, such that the following LMI is satisfied:

$$
\left[\begin{array}{ll}
\mathcal{E} & -\mathcal{A}
\end{array}\right]^{\perp *} \Theta\left[\begin{array}{ll}
\mathcal{E} & -\mathcal{A}
\end{array}\right]^{\perp}>0
$$

where matrices $\mathcal{E}, \mathcal{A}$ and $\Theta$ are defined in (10), (11) and (12), then the system (1) is asymptotically stable for any constant gap $h \leq h_{\max }$.

Proof: It has been proved that using extended states $x_{k}$, the original system (1) could be modeled as in Figure 1 with the uncertain matrix $\nabla(s)$ defined in (9) and the linear transformation defined by (10), (11). Using Lemma 7, a separator $\Theta$ defined in (12) allows to satisfy the condition (3). Hence, Applying Theorem 1, the feedback system is well-posed if the first condition (2) is also satisfied with $\mathcal{E}$, $\mathcal{A}$ and $\Theta$ defined in (10), (11) and (12). Following therefore [2], it can be proved that the well-posedness of the feedback system implies that there do not exist eigenvalues of the distributed delay system on the right half plane, that is

$$
\begin{aligned}
& \forall h \leq h_{\max }, \forall s \in \mathbb{C}, R e(s) \geq 0, \\
& \operatorname{det}\left(s 1_{n}-A-A_{d} \int_{0}^{\infty} K(\theta) e^{-s \theta} e^{-s h} d \theta\right) \neq 0 .
\end{aligned}
$$

which concludes the proof.

Remark 1: The stability condition as stated in Theorem 2 is delay-dependent since it proves the stability of (1) for any constant gap $h$ lower than a prescribed upper bound $h_{\max }$. If the third operator $\left(1-e^{-s h}\right) / s$ in (9) is removed, adjusting correspondingly ${ }^{1}$ matrices $\mathcal{E}, \mathcal{A}$ and $\Theta$ in (10)-(11)-(12), an independent-of-delay (IOD) stability condition is provided.

Remark 2: Theorem 2 can be viewed as the robust approach counterpart of a Lyapunov-Krasovskii functional proposed recently in [12] defined by

$$
\begin{aligned}
V_{r}\left(x_{t}\right)= & \zeta_{r}^{T}(t) P \zeta_{r}(t)+h \int_{-h}^{0} \int_{t+\theta}^{t} \dot{x}^{T}(s) Q_{2} \dot{x}(s) d s d \theta \\
& +\int_{0}^{\infty} \int_{t-\frac{1}{\alpha} \theta-h}^{t} e^{-\theta} x^{T}(s) R_{1} x(s) d s d \theta
\end{aligned}
$$

where $P, Q_{2}, R_{1}$ are postive definite matrices and $\zeta_{r}(t)$ is an extended state which takes into account the projections of the state on Laguerre polynomials

$$
\zeta_{r}(t)=\left[\begin{array}{c}
x(t) \\
\int_{0}^{+\infty} L_{0}(\theta) x\left(t-\frac{1}{\alpha} \theta-h\right) e^{-\theta} d \theta \\
\vdots \\
\int_{0}^{+\infty} L_{r}(\theta) x\left(t-\frac{1}{\alpha} \theta-h\right) e^{-\theta} d \theta
\end{array}\right] .
$$

\section{NUMERICAL EXAMPLES}

\section{A. Example 1}

The model in this first example is extracted from [19], and represents the traffic flow dynamic of cars on a ring [13]. The system is expressed as (1) with

$$
A=\left[\begin{array}{cc}
-2 & 0 \\
0 & -2
\end{array}\right] \quad \text { and } \quad A_{d}=\left[\begin{array}{ll}
0 & 2 \\
2 & 0
\end{array}\right]
$$

\footnotetext{
${ }^{1}$ That is, removing the fourth and seventh rows from matrices $\mathcal{E}$ and $\mathcal{A}$, and removing the fourth column in $\mathcal{A}$ and the two rows/columns associated to the matrix $Q_{2}$ in the separator $\Theta$.
} 
TABLE I

STABILITY OF (13): MAXIMUM ALLOWABLE GAP.

\begin{tabular}{|c|c|l|}
\hline $\begin{array}{c}\text { sequence } \\
\text { order } r\end{array}$ & $\begin{array}{c}\text { maximum allowable } \\
\text { gap } h\end{array}$ & $\begin{array}{l}\text { remainder } \\
\text { bound } \lambda_{r}\end{array}$ \\
\hline 0 & 2.96 & $4.1 \times 10^{-5}$ \\
\hline 1 & 3.39 & $2.1 \times 10^{-7}$ \\
\hline 2 & 3.43 & $2.3 \times 10^{-9}$ \\
\hline 3 & 3.43 & $4.3 \times 10^{-11}$ \\
\hline
\end{tabular}

The kernel is a gamma distribution

$$
K(\theta)=\frac{\theta^{N-1} e^{-\frac{\theta}{T}}}{T^{N}(N-1) !},
$$

for a given $N$ and a given $T$. For $N=1$ and $N=2$, [19] has shown that the system is stable for $(T, h) \in[0,1000] \times$ $[0,1000]$. This result is recovered with Theorem 2 . It can be proved that it is actually IOD stable, for both $N=1$ and $N=2$, with a slight modification in Theorem 2 (see Remark 1 ). Note that for $N=1$, it is sufficient to set $r=0$ since in that case the function $f$ is constant: $f(\theta)=\frac{1}{T}$. When $N=2$, a zero $r$ is not enough for this example and a first order Laguerre polynomial $(r \geq 1)$ is necessary to prove the stability, the remainder operator bound $\lambda$ being too high otherwise. For instance, for $T=2$ and $h=100$, when $r=0$ the bound $\lambda$ equals 0.54 while it vanishes to 0 when $r=1$.

\section{B. Example 2}

Let consider the following scalar example:

$$
\dot{x}(t)=0.2 x(t)-5 \int_{0}^{\infty} \frac{1+\theta}{5+\theta} e^{-4 \theta} x(t-\theta-h) d \theta .
$$

Notice that removing the delayed part, the system is unstable. According to our modeling, we have $f(\theta)=\frac{1+\theta}{5+\theta}$ and $\alpha=4$. The function $f$ being a rational fraction, the projection on the Laguerre polynomials sequence won't be exact. Theorem 2 allows to establish the stability of (13) for any value of the gap $h$ lower than a certain upperbound as stated in Table I. One can observe that the higher the order $r$ of the Laguerre polynomial basis is, the lower the remainder bound $\lambda_{r}$ is. Hence, conservatism is reduced as $r$ increases and a larger maximum allowable gap is found.

\section{CONCLUSION}

In this paper, we have studied the stability of a class of distributed delay systems with infinite support. Unlike the classical approach based on the construction of Lyapunov functionals, we have transform the problem as a robust stability problem. Within this framework, using quadratic separation, all the distributed delay transfer functions are embedded into uncertainties. These uncertainties are then characterized by quadratic inequalities. To this end, we proposed to develop new inequalities based on the use of Laguerre polynomials and the Bessel-Laguerre inequality. This last inequality makes it possible to obtain LMI conditions whose conservatism depends on the chosen degree for the Laguerre polynomials.

\section{REFERENCES}

[1] Y. Ariba, F. Gouaisbaut, and A. Seuret. Bessel inequality for robust stability analysis of time-delay system. In 52nd IEEE Conference on Decision and Control (CDC'13), 2013.

[2] Y. Ariba, F. Gouaisbaut, A. Seuret, and D. Peaucelle. Stability analysis of time-delay systems via Bessel inequality: A quadratic separation approach. International Journal of Robust and Nonlinear Control, 28(5):1507-1527, 2018.

[3] W. Chen and W. Zheng. Delay-dependent robust stabilization for uncertain neutral systems with distributed delays. Automatica, 43(1):95 - 104, 2007.

[4] Z. Feng and J. Lam. Integral partitioning approach to robust stabilization for uncertain distributed time-delay systems. International Journal of Robust and Nonlinear Control, 22(6):676 - 689, 2012.

[5] E. Fridman. Introduction to Time-Delay Systems. Springer, 2014

[6] F. Gouaisbaut, Y. Ariba, and A. Seuret. Stability of distributed delay systems via a robust approach. In 2015 European Control Conference (ECC), pages 2068-2073, July 2015.

[7] F. Gouaisbaut and D. Peaucelle. Delay-dependent stability analysis of linear time delay systems. In IFAC workshop on time delay system, Aquila,Italy, 2006.

[8] K. Gu. An integral inequality in the stability problem of time-delay systems. IEEE Conference on Decision and Control, 2000.

[9] K. Gu, V.-L. Kharitonov, and J. Chen. Stability of time-delay systems. Birkhauser, 2003.

[10] T. Iwasaki and S. Hara. Well-posedness of feedback systems: insights into exact robustness analysis and approximate computations. IEEE Trans. on Automatic Control, 43(5):619-630, May 1998.

[11] K. Liu, E. Fridman, K. H. Johansson, and Y. Xia. Generalized jensen inequalities with application to stability analysis of systems with distributed delays over infinite time-horizons. Automatica, 69:222-231, 2016.

[12] K. Liu, A. Seuret, Y. Xia, F. Gouaisbaut, and Y. Ariba. Bessel-Laguerre inequality and its application to systems with infinite distributed delays. Submitted, 2018.

[13] C. Morarescu, S. Niculescu, and K. Gu. Stability crossing curves of shifted gamma-distributed delay systems. SIAM Journal on Applied Dynamical Systems, 6(2):475-493, 2007.

[14] D Peaucelle, D Arzelier, D Henrion, and F Gouaisbaut. Quadratic separation for feedback connection of an uncertain matrix and an implicit linear transformation. Automatica, 43(5):795-804, 2007.

[15] A. Seuret and F. Gouaisbaut. Wirtinger-based integral inequality: Application to time-delay systems. Automatica, 49(9):2860 - 2866, 2013.

[16] A. Seuret and F. Gouaisbaut. Hierarchy of LMI conditions for the stability analysis of time-delay systems. Systems \& Control Letters, $81: 1-7,2015$

[17] A. Seuret, F. Gouaisbaut, and Y.Ariba. Complete quadratic Lyapunov functionals for distributed delay systems. Automatica, 62:168 - 176, 2015.

[18] R. Sipahi, F. M. Atay, and S. I. Niculescu. Stability of traffic flow behavior with distributed delays modeling the memory effects of the drivers. SIAM Journal on Applied Mathematics, 68(3):738-759, 2007.

[19] O. Solomon and E. Fridman. New stability conditions for systems with distributed delays. Automatica, 49(11):3467 - 3475, 2013.

[20] E. Witrant, E. Fridman, O.Sename, and L. Dugard, editors. Recent Results on Time-Delay Systems. Number 5 in Advances in Delays and Dynamics. Springer, 2016.

[21] H.B. Zeng, Y. He, M. Wu, and J. She. New results on stability analysis for systems with discrete distributed delay. Automatica, 60:189 - 192, 2015.

[22] J. Zhang, C. R. Knopse, and P. Tsiotras. Stability of time-delay systems: Equivalence between Lyapunov and scaled small-gain conditions. IEEE Trans. on Automatic Control, 46(3):482-486, March 2001. 